\section{Mitteilung aus dem Berufsverband}

\author{
Kommentar zum BGH-Urteil vom 19. 11. 1997: \\ Ärztliche Falschbehandlung bei der Strahlentherapie
}

Bezug: Aufklärungspflicht bei vertretungsweiser Fortführung einer Strahlentherapie (Strahlenther Onkol 1998;174:446)

1. Die Behandlung mit Gammastrahlen in therapeutisch wirksamer Dosis stellt einen erheblichen Eingriff in die körperliche Unversehrtheit dar. Die Einwilligung des Patienten rechtfertigt bei Fehlen einer weitergehenden Aufklärung nur eine lege artis durchgeführte Strahlentherapie.

2. Ein ärztlicher Urlaubsvertreter darf eine von dem vertretenen Arzt begonnene Therapie nach dessen Bestrahlungsplan jedenfalls dann nicht ungeprüft weiterführen, wenn ausreichende Anhaltspunkte für ernste Zweifel an dessen Richtigkeit für ihn erkennbar sind.

BGH-Urteil vom 19.11.997 - 3 StR 271/97 (LG Düsseldorf)

Dies sind die letzten Versuche, aus dem Verfahren zu Hilden bei Düsseldorf gut herauszukommen. Sie sind vergebens gewesen. Bedeutsam ist, daß sich der Angeklagte nicht darauf berufen konnte, er habe die Mängel der Strahlentherapie des Hauptschuldigen deshalb nicht erkannt, weil er seinerseits mangels Fortbildung den aktuellen Stand der Strahlentherapie nicht gekannt habe. Denn ein Arzt ist verpflichtet, sich in dem Umfang fortzubilden, wie es zur Erhaltung und Entwicklung der $\mathrm{zu}$ seiner Berufsausübung erforderlichen Fachkenntnisse notwendig ist. Demnach muß also auch ein ärztlicher Urlaubsvertreter über die Fachkenntnisse verfügen, die ihn befähigen, die bei der Vertretung vorgenommenen Behandlungen lege artis durchzuführen. Es ist in der Rechtsprechung anerkannt, $\mathrm{da} B$ auch derjenige schuldhaft handeln kann, der eine Tätigkeit vornimmt, obwohl er weiß oder erkennen kann, daß ihm die dafür erforderlichen Kenntnisse fehlen. Das Verschulden kann sowohl in der Übernahme einer die Fähigkeiten des Handeln übersteigenden Tätigkeit als auch in ihrer Fortführung liegen.

Hans-Bruno Makoski, Duisburg

\section{Preisausschreibung}

Die Vereinigung Deutscher Strahlenschutzärzte e. V. und die Hanns-Langendorff-Stiftung verleihen anläßlich der Jahrestagung der Vereinigung Deutsche Strahlenschutzärzte vom 29. 4. bis 1. 5. 1999 in Witten/Herdecke zur Förderung des Strahlenschutzes erneut den Hanns-Langendorff-Preis. Der Preis ist mit DM $10000,-$ dotiert und soll an jüngere Wissenschaftler verliehen werden, die sich durch eine oder mehrere hervorragende Arbeiten auf dem Gebiet des medizinischen Strahlenschutzes verdient gemacht haben.

Förderungswürdig sind Arbeiten, die sich mit den strahlenbiologischen und physikalischen Grundlagen sowie dem angewandten Strahlenschutz in Röntgendiagnostik, Nuklearmedizin und Strahlentherapie befassen. Der Preis ist teilbar.

Einsendungen von Arbeiten der Bewerber müssen in sechs Exemplaren bis zum 1. 2. 1999 erfolgen und sind zu richten an den Vorsitzenden der Vereinigung Deutscher Strahlenschutzärzte e. V. Herrn Dr. K. G. Hering, Knappschaftskrankenhaus, Radiologische Klinik Wieckesweg 27, D-44309 Dortmund, e-mail: K.G.Hering@t-online.de

\section{DEGRO-News}

\author{
4. Deutscher Kongreß für Radioonkologie, \\ Strahlenbiologie und Medizinische Physik, \\ Nürnberg 7. bis 10. November 1998
}

Der 4. Kongreß der Deutschen Gesellschaft für Radioonkologie (DEGRO) findet vom 7. bis 10. November 1998 im Messezentrum Nürnberg statt. Der Kongreßpräsident ist Prof. Dr. med. Rolf Sauer, Direktor der Klinik für Strahlentherapie der Universität Erlangen-Nürnberg.

Auch in diesem Jahr liegt der Schwerpunkt des Kongresses in der Darstellung neuer Entwicklungen in klinischer Wissenschaft, Grundlagenforschung und Technik. In enger Kooperation mit der Deutschen Gesellschaft für Medizinische Physik, der Deutschen Gesellschaft für Biologische Strahlenforschung und der Deutschen Gesellschaft für Nuklearmedizin werden wiederum interessante Themen aus den jeweiligen Fachrichtungen behandelt.

Für Symposien zu Themen allgemeinen Interesses (zum Beispiel Mammakarzinom, kolorektale Tumoren, prädiktive Tests, PET in der Onkologie, molekularbiologische Techniken in der radioonkologischen Grundlagenforschung) wurden namhafte natio-nale und internationale Referenten gewonnen, die einen Überblick über den jeweiligen Themenkomplex vermitteln werden.

Für Anfänger zum Einstieg und für Erfahrene zur Repetition werden Refresherkurse angeboten. Neben klinischen Themen und solchen aus der Biologie und Biostatistik wurde ein neuer Schwerpunkt mit dem Gebiet Onkodiagnostik (unter der Leitung von Prof. Dr. P. Lukas, Innsbruck) gesetzt.

Am 7. und 8. November werden erneut Fortbildungsseminare für medizinisch-technische Radiologieassistenten und Pflegekräfte in der Onkologie angeboten.

Ein attraktives Rahmen- und Kulturprogramm erschwert dem Kongreßteilnehmer die Entscheidung zwischen professioneller und privater Fortbildung, macht den Kongre $ß$ jedoch um so reizvoller für Begleitpersonen.

\section{Grenzenloses Interesse am Thema Strahlentherapie: LKW mit Infostand geklaut}

Schon immer wurde der Krebskongreß zahlenmäßig von den chemotherapeutisch tätigen Disziplinen dominiert. Vor allem in der Industrieausstellung waren nahezu ausschließlich die Pharmafirmen vertreten. So konnte der nicht spezialisierte Besucher den Eindruck gewinnen, Tumortherapie gleich Chemotherapie. Gegen dieses Informationsdefizit wollte die DEGRO gemeinsam mit der ARO und dem ZVEI (Zentralverband der Elektroindustrie in der Medizin) etwas unternehmen: Auf dem 23. Krebskongreß sollte ein firmenübergreifender Stand der drei Gesellschaften unter Beteiligung der Gerätehersteller Elektra, Siemens und Nucletron mit Video, Posterwänden und Broschüren den Interessierten über Grundsätzliches und Neues in der Strahlentherapie informieren.

Am Vorabend der Eröffnung des Krebskongresses reiste ein LKW mit sämtlichen Informationsmaterialien nach Berlin. Kurz darauf war das Fahrzeug samt Inhalt verschwunden und wurde nie wieder gesehen. Sicher wurden die Diebe herb enttäuscht, fanden sie doch statt der erhofften elektronischen Geräte nur Plakatwände und viel Papier.

Ein Trost war, daß der daraufhin eilig improvisierte Ersatzstand zwar vom Layout nicht ganz so ansprechend war wie das geplante Original, dafür aber umso lebhafteren Andrang fand.

Vermutungen einiger internistischer Kollegen, es habe sich hierbei um einen Publicity-Gag gehandelt, weisen die Veranstalter mit Entschiedenheit zurück!

M.-L. Sautter-Bihl 